\title{
Fungicide-induced hormesis in phytopathogenic fungi: A critical determinant of successful agriculture and environmental sustainability
}

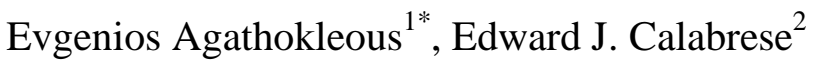

\begin{abstract}
${ }^{1}$ Key Laboratory of Agrometeorology of Jiangsu Province, Department of Ecology, School of Applied Meteorology, Nanjing University of Information Science \& Technology, Nanjing 210044, China.

${ }^{2}$ Department of Environmental Health Sciences, Morrill I, N344, University of Massachusetts, Amherst, MA 01003, USA.

*Correspondence and requests for materials should be addressed to E.A. (evgenios@nuist.edu.cn).
\end{abstract}

\section{Reference support and further detailed discussion of the supporting literature}

\section{Background (hormesis)}

Dose-response relationship holds a central role in ecotoxicology, (environmental) toxicology, risk assessment, and ecology to e.g. identify toxicity thresholds, derive critical levels of pollutants, study biological plasticity, and rank species/organisms tolerance or susceptibility. 1-6 Hormesis is a biphasic dose response (dose refers to dose or concentration hereafter), encompassing the full spectrum of the dose-response relationship, including biologically important responses at both below (hereafter low doses) and above (hereafter high doses) the noobserved-adverse-effect level (NOAEL) (Figure 1). ${ }^{1-6}$ Massive extensive evaluations of the published literature have recently demonstrated that numerous environmental stresses, including global pollutants and emerging contaminants, commonly induce hormesis in algae, bacteria, fungi, plants (and fresh fruits and vegetables), and other living organisms. ${ }^{5-15}$ For example, herbicides, e.g. glyphosate and 2,4-Dichlorophenoxyacetic acid (2,4-D), have been widely shown to induce hormesis in diverse types of plants. ${ }^{16-19}$ In these cases, herbicides not only do not cause toxicities to plants, but they rather stimulate plant performance at low doses. ${ }^{16-19}$ Importantly, low-dose stimulatory responses activate adaptive response mechanisms that prepare organisms for more intense stress, thus being "stronger" and performing better in subsequent otherwise 
harmful exposures. ${ }^{10,20,21}$ Furthermore, the low-dose stimulatory responses can eventually lead to the development of resistance to environmental challenges, such as antimicrobial resistance in microbes or herbicide resistance in plants, which may act as a driver of evolution. ${ }^{10,17,22-24}$ These new understandings came to revolutionize stress biology, ecological risk assessment, and environmental impact assessments. ${ }^{1,4,25,26}$

\section{Recent evidence (detailed discussion of each study's findings)}

Important evidences have recently accumulated showing that fungicides induce hormesis in phytopathogenic fungi. A recent high-resolution study included multiple independent trials to study hormetic responses of Sclerotinia sclerotiorum, a notorious pathogen of over 400 plant species, to boscalid, a synthetic carboxamide fungicide. ${ }^{27}$ Two dimethachlone-resistant isolates (HLJ3 and HLJ4) and two dimethachlone-sensitive isolates (HLJMG1 and JD391) of S. sclerotiorum were studied. When boscalid was added into potato dextrose agar (PDA) at doses in the range $0.0005-0.002 \mu \mathrm{g} \mathrm{mL}^{-1}$, it significantly stimulated mycelial growth of four isolates of $S$. sclerotiorum (the maximum stimulation, MAX, was $6 \%$ on average). While at $0.02 \mu \mathrm{g} \mathrm{mL} \mathrm{m}^{-1}$ boscalid inhibited the mycelial growth of the dimethachlone-resistant isolates in PDA, inoculation of the inhibited mycelia on rapeseed leaves revealed that the two isolates displayed enhanced virulence (9 and 18\%). ${ }^{27}$ A further assay with sprayed boscalid (0.0001-0.1 $\mu \mathrm{g} \mathrm{mL}^{-1}$ ) on detached leaves of rapeseed revealed significantly stimulated virulence in the four isolates of S. sclerotiorum; the MAX was on average $18 \%$. Interestingly, there was no significant effect on the expression levels of the virulence-associated genes that encode cutinase (SsCut), polygalacturonase (SsPG1), and oxaloacetate acetylhydrolase (SsOah1). ${ }^{27}$

In different studies with the widely used carbendazim (benomyl's metabolite), a systemic, broad-spectrum fungicide, spray application of $0.001-0.03 \mu \mathrm{g} \mathrm{mL}{ }^{-1}$ stimulated the virulence of isolates HB459 (MAX=16\%) and HB536 (MAX=21\%) of Botrytis cinerea, an airborne, necrotrophic pathogen of over 200 crops, especially wine grapes.$^{28}$ Similar to the study 
with boscalid-S. sclerotiorum, ${ }^{27}$ incorporation of $0.01,0.02$, and $0.05 \mu \mathrm{g} \mathrm{mL}{ }^{-1}$ carbendazim in PDA inhibited HB536 mycelial growth by 1, 10, and $31 \%$, respectively; however, inoculation of the inhibited mycelia on cucumber leaves stimulated virulence by 10,13 , and $11 \%$, respectively. Carbendazim at 0.005 and $0.02 \mu \mathrm{g} / \mathrm{ml}$ in PDA stimulated sclerotial production, while at $0.1 \mu \mathrm{g}$ $\mathrm{mL}^{-1}$ inhibited the sclerotial number and mass of both isolates. Moreover, carbendazim at 0.001 and $0.005 \mu \mathrm{g} \mathrm{mL}{ }^{-1}$ in PDA increased the rates of conidia germination. In a further trial, 0.001$0.02 \mu \mathrm{g} \mathrm{mL}{ }^{-1}$ carbendazim cosuspended with conidia or sprayed on cucumber leaves stimulated the virulence of $B$. cinerea conidia. ${ }^{28}$ Interestingly, sub-NOAEL carbendazim doses of carbendazim did not significantly enhance the expression levels of Bcpg2 (endopolygalacturonas), Bcpme1 (pathogenicity-related pectin methylesterase gene), CutA (cutinase), BcnoxA (NADPH oxidase), and Xyn11A (cuxylanase) genes. ${ }^{28}$

Spraying flusilazole $(0.1 \mu \mathrm{g} / \mathrm{ml})$, a demethylation-inhibitor (DMI) fungicide, also stimulated the virulence of the notorious S. sclerotiorum, which was inoculated 1 and $24 \mathrm{~h}$ following the spraying. ${ }^{29}$ At $10 \mu \mathrm{g} / \mathrm{ml}$, however, it inhibited the virulence of S. sclerotiorum, which was inoculated $3 \mathrm{~d}$ following the spraying, while no significant effects were found when inoculated 5, 7, or $10 \mathrm{~d}$ following spraying. Preconditioning on flusilazole-treated (0.0005-0.25 $\mu \mathrm{g} / \mathrm{ml}$ ) PDA for $2 \mathrm{~d}$ and transfer of mycelia on fungicide-free PDA led to slower mycelia growth compared to non-preconditioned group. Conversely, transfer of preconditioned colonies on flusilazole-amended $(0.2 \mu \mathrm{g} / \mathrm{ml})$ PDA led to biphasic/parabolic response of mycelia growth to flusilazole preconditioning dose range, a dose-response relationship that was similar to that of mycelial growth of the preconditioned mycelial plugs on PDA treated with DMI fungicides prochloraz and tebuconazole (but not carbendazim or dimethachlone). ${ }^{29}$ In a further assay, flusilazole-preconditioned S. sclerotiorum on rapeseed plants displayed dose-dependent enhancement of virulence, response patterns that were similar with those observed on PDA. After disease lesions developed on flusilazole-preconditioned rapeseed leaves were inoculated on 
rapeseed plants, enhanced virulence was observed on leaves sprayed with prochloraz, flusilazole, or tebuconazole, relative to the nonpreconditioned group, while decreased virulence was observed on fungicide-free leaves or leaves treated with dimethachlone or carbendazim. In all these assays included in this integrated study, MAX was always smaller than $20 \%$ above the control response.

Another recent study evaluated hormetic responses of two dimethachlone-resistant isolates (JMS14 and HLJ4) of S. sclerotiorum to mixtures of dimethachlone and prochloraz in PDA. ${ }^{30}$ Dimethachlone stimulatory zone occurred in the range $1-100 \mu \mathrm{g} \mathrm{mL}{ }^{-1}$ for mycelia growth of the two isolates, and the doses of 10 and $50 \mu \mathrm{g} \mathrm{mL} \mathrm{m}^{-1}$ produced MAXs of 81 and $19 \%$ in JMS14 and HLJ4. Prochloraz, an imidazole fungicide introduced in the late 1970s, at 0.0003 and $0.002 \mu \mathrm{g} \mathrm{mL}^{-1}$ produced MAXs of 9 and $11 \%$ in JMS14 and HLJ4. Interestingly, the mixture of the two fungicides at their respective stimulatory doses produced MAXs of 48 and 9\% in JMS14 and HLJ4. When the mycelia with inhibited growth were subcultured on fungicide-free PDA, the second generation exhibited enhanced mycelia growth. ${ }^{30}$ Besides, inoculation of mycelia from fungicide-treated PDA on rapeseed leaves a considerably larger stimulation of virulence was observed (compared to fungicide-free PDA), while the inhibited mycelia displayed enhanced virulence on leaves. Likewise, mixture of prochloraz $\left(0.03 \mu \mathrm{g} \mathrm{mL}^{-1}\right)$ and dimethachlone $(100 \mu \mathrm{g}$ $\mathrm{mL}^{-1}$ ) inhibited JMS14 mycelia growth by $60 \%$ in PDA, but virulence was enhanced by $69 \%$ when the inhibited mycelia were inoculated on rapeseed leaves. A further assay with sprayed sub-NOAEL doses of prochloraz and dimethachlone on rapeseed leaves reproduced significant stimulation of virulence, and dose-additive interactions regarding virulence stimulation were found. ${ }^{30}$ Single prochloraz spray $\left(10-50 \mu \mathrm{g} \mathrm{mL} \mathrm{m}^{-1}\right)$ inhibited virulence, whereas single dimethachlone spray (10-50 $\left.\mu \mathrm{g} \mathrm{mL} \mathrm{m}^{-1}\right)$ significantly stimulated virulence. ${ }^{30}$ Their mixture $(1: 1$ ratio) led to higher inhibition than that of prochloraz alone. The two fungicides alone and in combination enhanced the mycelia tolerance to hydrogen peroxide $\left(\mathrm{H}_{2} \mathrm{O}_{2}\right)$. Prochloraz decreased 
but dimethachlone increased the membrane permeability of mycelia cell, while their mixture had additive interactions regarding cell membrane permeability. ${ }^{30}$

A different high-resolution study evaluated hormesis in isolates of Globisporangium ultimum and G. irregulare (oomycetal species) that are resistant to mefenoxam, an acylalanine fungicide with systemic function, by exposing them to ten concentrations of mefenoxam (0.01$\left.1,000 \mu \mathrm{g} \mathrm{mL} L^{-1}\right) .{ }^{31}$ The two species displayed hormesis with significant stimulatory responses of mycelia total growth area and total dry mass (MAX $<20 \%$ for all species and endpoints). ${ }^{31}$

In further extensive evaluations (not cited in the main manuscript due to space and reference limitations), 28 isolates of Sclerotinia homoeocarpa, causing dollar spot disease in turf, were cultured in corn meal agar containing 11 different concentrations $\left(0.047-30,500 \mu \mathrm{g} \mathrm{mL}^{-1}\right)$ of thiophanate-methyl (T-methyl), used for preventing and treating against a wide range of diseases, in direct exposures or using a preconditioning protocol (i.e. exposure following a preceding exposure to T-methyl). ${ }^{32}$ Notably, the mycelia growth of 17 (81\%) preconditioned T-methyltolerant isolates was significantly enhanced by 3-20\%. Reference isolates with no previous Tmethyl exposure were not stimulated by low T-methyl doses. ${ }^{32}$ Two sensitive isolates preconditioned with T-methyl and four T-methyl-tolerant isolates did not show significant stimulation of mycelia growth too. These findings are of considerable importance since they show that hormesis is reproducible and commonly occurs in preconditioned fungi.

Earlier evidence supporting this discussion (references could not be cited in the main text due to the number of allowed references limited to five)

The recent, important evidence of fungicide-induced hormesis in fungi found in the five cited studies with multi-factorial experimental designs, e.g. involving a number of independent assays, different species, and/or various isolates, finds support to further extensive evidence of fungicide-induced hormesis in fungi and oomycetes previously reported in the published literature. ${ }^{33,34}$ For example, a study revealed that 3 (out of 3 tested; 100\%) dimethachlon-resistant 
isolates and 13 (out of 55 isolates tested; 24\%) dimethachlon-sensitive isolates of S. sclerotiorum presented significant sub-NOAEL stimulation, while dimethachlon-resistant isolates grew faster on dimethachlon-treated $\left(0.5-4 \mu \mathrm{g} \mathrm{mL}^{-1}\right)$ PDA than on dimethachlon-free PDA. ${ }^{35}$ Dimethachlonresistant isolates cultured on dimethachlon-treated $\left(0.5-2 \mu \mathrm{g} \mathrm{mL}^{-1}\right)$ PDA displayed larger lesion diameters on detached leaves of oilseed rape plants, suggesting enhanced virulence. The MAXs revealed in the study were ranged from 42 to $60 \%$. An additional pot experiment with both dimethachlon-sensitive and -resistant isolates revealed that a spray application of $2 \mu \mathrm{g} \mathrm{mL} \mathrm{m}^{-1}$ dimethachlon significantly stimulated virulence. ${ }^{35}$ Based on an assay with dimethachlonamended PDA, the sensitivity of $S$. sclerotiorum to $\mathrm{H}_{2} \mathrm{O}_{2}$ decreased significantly (compared to dimethachlon-free PDA), ${ }^{35}$ which is in agreement with the study reporting enhanced tolerance of S. sclerotiorum mycelia to $\mathrm{H}_{2} \mathrm{O}_{2}$ after exposure to dimethachlone and prochloraz alone or in combination. ${ }^{30}$ Hence, these study show the potential of hormetic sub-NOAEL stimulation to considerably decrease the sensitivity of fungi to further environmental challenges, which may have further implications to plant disease management and ecological health. ${ }^{36}$ In a different study, hormetic responses were found in the soilborne, plant-pathogen oomycytes Pythium aphanidermatum (strain P18) exposed to cyazofamid and propamocarb, with a modeled MAX smaller than $20 \%$ (based on three repetitions of the experiment). ${ }^{37}$ The strain used was described resistant to mefenoxam and propamocarb and found to show hormetic response to mefenoxam, leading to increased mean radial growth and higher rates of Pythium damping-off of geranium plants. ${ }^{38,39}$ The MAX revealed was no greater than $\approx 60 \%$, but the amplitude varied depending on the assay/endpoint and time point. ${ }^{39}$ Collectively, various phytopathogens of high importance in agriculture have been found to exhibit fungicide-induced hormesis, including endpoints of pathogenicity.

Apart from the accumulated evidence of hormesis in phytopathogens with relevance to agriculture, it should be clarified that there is also extensive evidence for hormesis induced by 
diverse chemicals in various types of microorganisms ${ }^{40,41}$, including yeast ${ }^{42}$, which is of relevance to food systems and human health. Importantly, a study also found hormesis in various strains of Agaricus bisporus, an edible basidiomycete mushroom, exposed to three fungicides separately or all in combination (carbendazim (benzimidazol), tebuconazole (triazole), and kresoxim methyl (strobilurin)).$^{43}$ With the exception of carbendazim alone, the other chemicals alone or all together induced hormesis in mycelia growth or mushroom yield, with a MAX $<50 \%$; however, the presence of hormesis varied among isolates, applied chemicals, endpoints, and experiments. While this study did not aim at identifying the MAX, thus including only a limited number of sub-NOAEL doses, it shows that mixtures of fungicides can induce significant hormesis, ${ }^{43}$ in agreement with the findings of the study exposing S. sclerotiorum to mixtures of dimethachlone and prochloraz in PDA. ${ }^{30}$

The MAX was commonly up to $\approx 60 \%$ greater than the control (fungicide-free growing medium), and as a rule within two-fold the control response. These estimates are in good agreement with the broad hormesis literature for plants, microorganisms, and animals, ${ }^{12,25,40-42}$ albeit the MAX is known to positively associate with the number of doses included in the lowdose zone of the dose-response relationship. ${ }^{40}$ Hence, more studies should consider incorporating a higher number of sub-NOAEL doses (e.g. 12-15) to confirm whether the fungicide-induced MAX in phytopathogenic fungi and oomycytes can well exceed two-fold the control response. The existing studies also provide dose-response relationships indicating that the MAX-toNOAEL distance ranges from less than 2-fold to about 40-fold, depending on the assay, isolate, fungicide, and endpoint measured. ${ }^{27,28,30-32,35,37}$ This is important as it suggests that the doserange separating toxicity from maximum stimulation varies considerably; however, the underpinning mechanisms of this phenomenon are yet to be revealed. 
While the molecular mechanisms behind fungicide-induced hormesis are poorly understood, recent research indicates that sub-NOAEL fungicide exposure may increase mutation rates in fungi. ${ }^{33}$

Treatment with fungicides at doses lower than the (inhibitory) recommended may enhance the pathogenicity of target (or non-target) species and make the situation worse for the farmers rather than better. This is a similar situation for the cancer area, when the drug used to kill a tumor enhances the proliferation at low doses. ${ }^{44}$

Hormesis can be induced by fungicides in other types of fungi that are non-target species but critical determinants of agricultural productivity as well as ecological health. For example, a study published very recently showed that fenpropimorph (a morpholine-derived fungicide applied in the agricultural practice, primarily on cereal crops) induced hormesis, with low-dose stimulation and high-dose inhibition, in the hyphal healing mechanism of Rhizophagus irregularis, an arbuscular mycorrhizal species inoculated in soil in agricultural and horticultural practice. $^{45}$

\section{References}

(1) Agathokleous, E.; Calabrese, E. J. Environmental Toxicology and Ecotoxicology: How Clean Is Clean? Rethinking Dose-Response Analysis. Sci. Total Environ. 2020, 746, 138769. https://doi.org/10.1016/j.scitotenv.2020.138769.

(2) Agathokleous, E.; Saitanis, C. J. Plant Susceptibility to Ozone: A Tower of Babel? Sci. Total Environ. 2020, 703, 134962. https://doi.org/10.1016/j.scitotenv.2019.134962.

(3) Agathokleous, E.; Belz, R. G.; Calatayud, V.; De Marco, A.; Hoshika, Y.; Kitao, M.; Saitanis, C. J.; Sicard, P.; Paoletti, E.; Calabrese, E. J. Predicting the Effect of Ozone on Vegetation via Linear Non-Threshold (LNT), Threshold and Hormetic Dose-Response Models. Sci. Total Environ. 2019, 649, 61-74. 
https://doi.org/10.1016/j.scitotenv.2018.08.264.

(4) Agathokleous, E.; Anav, A.; Araminiene, V.; De Marco, A.; Domingos, M.; Kitao, M.; Koike, T.; Manning, W. J.; Paoletti, E.; Saitanis, C. J.; et al. Commentary: EPA’s Proposed Expansion of Dose-Response Analysis Is a Positive Step towards Improving Its Ecological Risk Assessment. Environ. Pollut. 2019, 246, 566-570. https://doi.org/10.1016/j.envpol.2018.12.046.

(5) Agathokleous, E.; Barceló, D.; Tsatsakis, A.; Calabrese, E. J. Hydrocarbon-Induced Hormesis: 101 Years of Evidence at the Margin? Environ. Pollut. 2020, 265, 114846. https://doi.org/10.1016/j.envpol.2020.114846.

(6) Calabrese, E. Hormesis: Why It Is Important to Toxicology and Toxicologists. Environ. Toxicol. Chem. 2008, 27 (7), 1451-1474. https://doi.org/10.1897/07-541.

(7) Agathokleous, E.; Feng, Z.; Iavicoli, I.; Calabrese, E. J. The Two Faces of Nanomaterials: A Quantification of Hormesis in Algae and Plants. Environ. Int. 2019, 131, 105044. https://doi.org/10.1016/j.envint.2019.105044.

(8) Agathokleous, E.; Kitao, M.; Calabrese, E. J. Hormetic Dose Responses Induced by Lanthanum in Plants. Environ. Pollut. 2019, 244, 332-341. https://doi.org/10.1016/j.envpol.2018.10.007.

(9) Agathokleous, E.; Araminiene, V.; Belz, R. G.; Calatayud, V.; De Marco, A.; Domingos, M.; Feng, Z.; Hoshika, Y.; Kitao, M.; Koike, T.; et al. A Quantitative Assessment of Hormetic Responses of Plants to Ozone. Environ. Res. 2019, 176, 108527. https://doi.org/10.1016/j.envres.2019.108527.

(10) Agathokleous, E.; Calabrese, E. J. A Global Environmental Health Perspective and Optimisation of Stress. Sci. Total Environ. 2020, 704, 135263. https://doi.org/10.1016/j.scitotenv.2019.135263.

(11) Calabrese, E. J.; Agathokleous, E. Accumulator Plants and Hormesis. Environ. Pollut. 
2021, 274. https://doi.org/10.1016/j.envpol.2021.116526.

(12) Shahid, M.; Niazi, N. K.; Rinklebe, J.; Bundschuh, J.; Dumat, C.; Pinelli, E. Trace Elements-Induced Phytohormesis: A Critical Review and Mechanistic Interpretation. Crit. Rev. Environ. Sci. Technol. 2020, 50 (19), 1984-2015. https://doi.org/10.1080/10643389.2019.1689061.

(13) Carvalho, M. E. A.; Castro, P. R. C.; Azevedo, R. A. Hormesis in Plants under Cd Exposure: From Toxic to Beneficial Element? J. Hazard. Mater. 2020, 384, 121434. https://doi.org/10.1016/j.jhazmat.2019.121434.

(14) Cisneros-Zevallos, L.; Jacobo-Velázquez, D. A. Controlled Abiotic Stresses Revisited: From Homeostasis through Hormesis to Extreme Stresses and the Impact on Nutraceuticals and Quality during Pre- and Postharvest Applications in Horticultural Crops. J. Agric. Food Chem. 2020, 68 (43), 11877-11879.

https://doi.org/10.1021/acs.jafc.0c06029.

(15) Duarte-Sierra, A.; Tiznado-Hernández, M. E.; Jha, D. K.; Janmeja, N.; J., A. Abiotic Stress Hormesis: An Approach to Maintain Quality, Extend Storability, and Enhance Phytochemicals on Fresh Produce during Postharvest. Compr. Rev. Food Sci. Food Saf. 2020, 19 (6), 3659-3682. https://doi.org/10.1111/1541-4337.12628.

(16) Agathokleous, E.; Feng, Z. Z.; Calabrese, E. J. The Systemic Herbicide 2,4Dichlorophenoxyacetic Acid (2,4-D) Is Another Hormetin: What Does It Mean for Agriculture and the Environment? J. Agric. Food Chem. 2019, 67 (35), 9695-9696. https://doi.org/10.1021/acs.jafc.9b04757.

(17) Belz, R. G.; Duke, S. O. Herbicides and Plant Hormesis. Pest Manag. Sci. 2014, 70 (5), 698-707. https://doi.org/10.1002/ps.3726.

(18) Brito, I. P.; Tropaldi, L.; Carbonari, C. A.; Velini, E. D. Hormetic Effects of Glyphosate on Plants. Pest Manag. Sci. 2018, 74 (5), 1064-1070. https://doi.org/10.1002/ps.4523. 
(19) Duke, S. O. Glyphosate: Environmental Fate and Impact. In Weed Science; Cambridge University Press, 2020; Vol. 68, pp 201-207. https://doi.org/10.1017/wsc.2019.28.

(20) Agathokleous, E.; Kitao, M.; Calabrese, E. J. Hormesis: A Compelling Platform for Sophisticated Plant Science. Trends Plant Sci. 2019, 24 (4), 318-327. https://doi.org/10.1016/j.tplants.2019.01.004.

(21) Calabrese, E. J. Preconditioning Is Hormesis Part II: How the Conditioning Dose Mediates Protection: Dose Optimization within Temporal and Mechanistic Frameworks. Pharmacol. Res. 2016, 110, 265-275. https://doi.org/10.1016/j.phrs.2015.12.020.

(22) Calabrese, E. J.; Agathokleous, E. Theodosius Dobzhansky’s View on Biology and Evolution v.2.0: “Nothing in Biology Makes Sense except in Light of Evolution and Evolution’s Dependence on Hormesis-Mediated Acquired Resilience That Optimizes Biological Performance and Numerous Diverse Short . Environ. Res. 2020, 186. https://doi.org/10.1016/j.envres.2020.109559.

(23) Belz, R. G. Herbicide Hormesis Can Act as a Driver of Resistance Evolution in Weeds PSII-Target Site Resistance in Chenopodium album L. as a Case Study. Pest Manag. Sci. 2018, 74 (12), 2874-2883. https://doi.org/10.1002/ps.5080.

(24) Belz, R. G.; Farooq, M. B.; Wagner, J. Does Selective Hormesis Impact Herbicide Resistance Evolution in Weeds? ACCase-Resistant Populations of Alopecurus myosuroides Huds. as a Case Study. Pest Manag. Sci. 2018, 74 (8), 1880-1891. https://doi.org/10.1002/ps.4890.

(25) Agathokleous, E.; Kitao, M.; Calabrese, E. J. Hormesis: Highly Generalizable and beyond Laboratory. Trends Plant Sci. 2020, 25 (11), 1076-1086. https://doi.org/10.1016/j.tplants.2020.05.006.

(26) Agathokleous, E. The Rise and Fall of Photosynthesis: Hormetic Dose Response in Plants. J. For. Res. 2021, 32, 789-803. https://doi.org/10.1007/s11676-020-01252-1. 
(27) Hu, S.; Xu, Q.; Zhang, Y.; Zhu, F. Stimulatory Effects of Boscalid on Virulence of Sclerotinia sclerotiorum Indicate Hormesis May Be Masked by Inhibitions. Plant Dis. 2020, 104 (3), 833-840. https://doi.org/10.1094/pdis-07-19-1421-re.

(28) Cong, M.-L.; Zhang, B.; Zhang, K.; Li, G.; Zhu, F. Stimulatory Effects of Sublethal Doses of Carbendazim on the Virulence and Sclerotial Production of Botrytis cinerea. Plant Dis. 2019, 103 (9), 2385-2391. https://doi.org/10.1094/pdis-01-19-0153-re.

(29) Lu, X.; He, S.; Ma, H.; Li, J.; Zhu, F. Hormetic Effects of Flusilazole Preconditioning on Mycelial Growth and Virulence of Sclerotinia sclerotiorum. Plant Dis. 2018, 102 (6), 1165-1170. https://doi.org/10.1094/pdis-10-17-1638-re.

(30) Zhang, R.; Zhang, Y.; Xu, Q.; Li, J.; Zhu, F. Hormetic Effects of Mixtures of Dimethachlone and Prochloraz on Sclerotinia sclerotiorum. Plant Dis. 2019, 103 (3), 546-554. https://doi.org/10.1094/pdis-06-18-1071-re.

(31) Pradhan, S.; Flores, F. J.; Molineros, J. E.; Walker, N. R.; Melouk, H.; Garzon, C. D. Improved Assessment of Mycelial Growth Stimulation by Low Doses of Mefenoxam in Plant Pathogenic Globisporangium Species. Eur. J. Plant Pathol. 2017, 147 (3), 477-487. https://doi.org/10.1007/s10658-016-1016-5.

(32) Pradhan, S.; Miller, L.; Marcillo, V.; Koch, A. R.; Graf Grachet, N.; Molineros, J. E.; Walker, N. R.; Melouk, H.; Garzon, C. D. Hormetic Effects of Thiophanate-Methyl in Multiple Isolates of Sclerotinia homoeocarpa. Plant Dis. 2019, 103 (1), 89-94. https://doi.org/10.1094/pdis-05-18-0872-re.

(33) Pesticide Dose: Effects on the Environment and Target and Non-Target Organisms; Duke, S. O., Kudsk, P., Solomon, K., Eds.; ACS Symposium Series; American Chemical Society: Washington, DC, 2017; Vol. 1249. https://doi.org/10.1021/bk-2017-1249.

(34) Garzon, C. D.; Flores, F. J. Hormesis: Biphasic Dose-Responses to Fungicides in Plant Pathogens and Their Potential Threat to Agriculture. In Fungicides - Showcases of 
Integrated Plant Disease Management from Around the World; InTech, 2013. https://doi.org/10.5772/55359.

(35) Zhou, F.; Liang, H. J.; Di, Y. L.; You, H.; Zhu, F. X. Stimulatory Effects of Sublethal Doses of Dimethachlon on Sclerotinia sclerotiorum. Plant Dis. 2014, 98 (10), 1364-1370. https://doi.org/10.1094/PDIS-10-13-1059-RE.

(36) Agathokleous, E.; Calabrese, E. J. Hormesis Can Enhance Agricultural Sustainability in a Changing World. Glob. Food Sec. 2019, 20 (C), 150-155.

https://doi.org/10.1016/j.gfs.2019.02.005.

(37) Flores, F. J.; Garzon, C. D. Detection and Assessment of Chemical Hormesis on the Radial Growth in Vitro of Oomycetes and Fungal Plant Pathogens. Dose-Response 2013, 11 (3), 361-373. https://doi.org/10.2203/dose-response.12-026.Garzon.

(38) Moorman, G. W.; Kim, S. H. Species of Pythium from Greenhouses in Pennsylvania Exhibit Resistance to Propamocarb and Mefenoxam. Plant Dis. 2004, 88 (6), 630-632. https://doi.org/10.1094/pdis.2004.88.6.630.

(39) Garzón, C. D.; Molineros, J. E.; Yánez, J. M.; Flores, F. J.; del Mar Jiménez-Gasco, M.; Moorman, G. W. Sublethal Doses of Mefenoxam Enhance Pythium Damping-off of Geranium. Plant Dis. 2011, 95 (10), 1233-1237. https://doi.org/10.1094/PDIS-09-100693.

(40) Calabrese, E. J.; Agathokleous, E.; Kozumbo, W. J. W. J.; Stanek, E. J. E. J. E. J.; Leonard, D. Estimating the Range of the Maximum Hormetic Stimulatory Response. Environ. Res. 2019, 170, 337-343. https://doi.org/10.1016/j.envres.2018.12.020.

(41) Calabrese, E. J.; Blain, R. B. The Hormesis Database: The Occurrence of Hormetic Dose Responses in the Toxicological Literature. Regul. Toxicol. Pharmacol. 2011, 61 (1), $73-$ 81. https://doi.org/10.1016/j.yrtph.2011.06.003.

(42) Calabrese, E. J. Hormesis Commonly Observed in the Assessment of Aneuploidy in 
Yeast. Environ. Pollut. 2017, 225, 713-728. https://doi.org/10.1016/j.envpol.2017.03.020.

(43) Zied, D. C.; Dourado, F. A.; Dias, E. S.; Pardo-Giménez, A. First Study of Hormesis Effect on Mushroom Cultivation. World J. Microbiol. Biotechnol. 2017, 33 (11), 195. https://doi.org/10.1007/s11274-017-2342-2.

(44) Calabrese, E. J. Cancer Biology and Hormesis: Human Tumor Cell Lines Commonly Display Hormetic (Biphasic) Dose Responses. Crit. Rev. Toxicol. 2005, 35 (6), 463-582. https://doi.org/10.1080/10408440591034502.

(45) Rodriguez-Morelos VH, Calonne-Salmon M, Bremhorst V, Garcés-Ruiz M and Declerck S. Fungicides With Contrasting Mode of Action Differentially Affect Hyphal Healing Mechanism in Gigaspora sp. and Rhizophagus irregularis. Front. Plant Sci. 2021, 12, 642094. https://doi.org/10.3389/fpls.2021.642094 